\title{
Свойства микрорезонатора на основе таммовского плазмона с внутрирезонаторными металлическими слоями и органической активной областью
}

\author{
(C) К.М. Морозов ${ }^{1,2}$, А.В. Белоновский ${ }^{1,2}$, Е.И. Гиршова ${ }^{1}$, К.А. Иванов ${ }^{2}$, М.А. Калитеевский ${ }^{1,2,3, \text { ฯ }}$ \\ ${ }^{1}$ Санкт-Петербургский Академический университет, \\ 194021 Санкт-Петербург, Россия \\ 2 Университет ИТМО, \\ 197101 Санкт-Петербург, Россия \\ ${ }^{3}$ Физико-технический институт им. А.Ф. Иоффе Российской академии наук, \\ 194021 Санкт-Петербург, Россия \\ `E-mail: kalit@mail.ioffe.ru
}

Поступила в Редакцию 19 сентября 2019 г.

В окончательной редакции 1 октября 2019 г.

Принята к публикации 5 ноября 2019 г.

\begin{abstract}
Проведено теоретическое исследование свойств микрорезонаторной структуры с внутрирезонаторными металлическими слоями и органической активной областью (малой молекулы CBP $\left(4,4^{\prime}\right.$-Bis $(N$-carbazolyl)-1,1'-biphenyl)). Структура состояла из слоя органического материала CBP заданной толщины, заключенного между двумя слоями серебра, тонкими фазосогласующими слоями СВР $\left(d_{\mathrm{ph}}=26\right.$ нм$)$ и двумя четвертьволновыми брэгговскими отражателями. Было показано, что гибридные моды, локализованные в центральном слое структуры, могут значительно увеличивать скорость затухания люминесценции органического излучателя посредством эффекта Парселла. Был обнаружен сдвиг гибридных мод при их взаимодействии с экситонным резонансом в СВР, что может означать о появлении режима сильной связи.
\end{abstract}

Ключевые слова: таммовский плазмон, органический светодиод, вертикально-излучающий лазер, экситон.

DOI: $10.21883 /$ FTP.2020.03.49033.9260

\section{1. Введение}

Органические светоизлучающие материалы на сегодняшний день широко используются для создания дисплеев, светодиодов и других приборов [1]. Для создания высокоэффективных источников света нового поколения требуется усиление излучательной рекомбинации и увеличение квантового выхода органических материалов, что в особенности касается материалов, излучающих в синем диапазоне [2].

Перспективным решением является использование резонансных плазмонных систем. Можно выделить структуры, поддерживающие таммовский плазмон - локализованное состояние на границе брэгговского отражателя и металла. Источники [3,4] и детекторы света [5-7] на основе таммовских плазмонов интенсивно исследуются в последнее время. Недавно было продемонстрировано увеличение скорости спонтанной эмиссии в структурах с таммовским плазмоном [8]. Недавно был предложен дизайн планарного металл-диэлектрического микрорезонатора с внутрирезонаторными металлическими слоями, поддерживающего гибридные таммовские плазмоны [9], перспективного для создания вертикально-излучающих лазеров [10], ввиду особенного распределения электрического поля моды. Было экспериментально продемонстрировано уменьшение поглощения в металлических слоях в таких системах по сравнению с обычным таммовским плазмоном [11].
Во многих органических светоизлучающих материалах экситоны обладают очень высокой силой осциллятора, что приводит к взаимодействию экситона в органическом материале и собственной оптической моды микрорезонатора в режиме сильной связи $[12,13]$.

В данной работе была теоретически исследована модовая структура металлодиэлектрического микрорезонатора с внутрирезонаторными металлическими слоями и органической активной областью, а также проанализировано взаимодействие между гибридной модой и экситоном в органическом материале СВР (4,4'-Bis $\left(N\right.$-carbazolyl)-1, $1^{\prime}$-biphenyl).

\section{2. Результаты и обсуждение}

Была рассмотрена планарная структура металлдиэлектрического микрорезонатора с внутрирезонаторными металлическими слоями. Структура состоит из слоя светоизлучающего органического материала СВР (4,4'-Bis $(N$-carbazolyl)-1,1'-biphenyl) [14] заданной толщины $\left(d_{\text {cav }}\right)$, заключенного между двумя слоями серебра толщинами 40 нм, тонкими фазосогласующими слоями СВР $\left(d_{\mathrm{ph}}=26 \mathrm{нм}\right)$ и двумя четвертьволновыми брэгговскими отражателями из слоев оксида кремния и оксида тантала с толщинами 66 и 43 нм соответственно. Материал СВР примечателен тем, что интерфейс между CВР и серебром поддерживает поверхностный плазмон с частотой, лежащей внутри полосы излучения СВР [15]. 


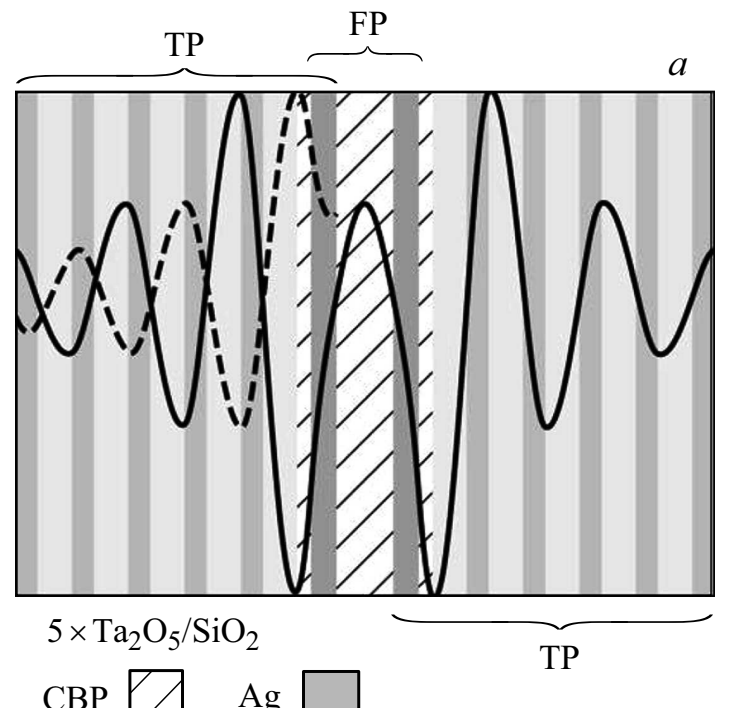

CBP Ag

$b$

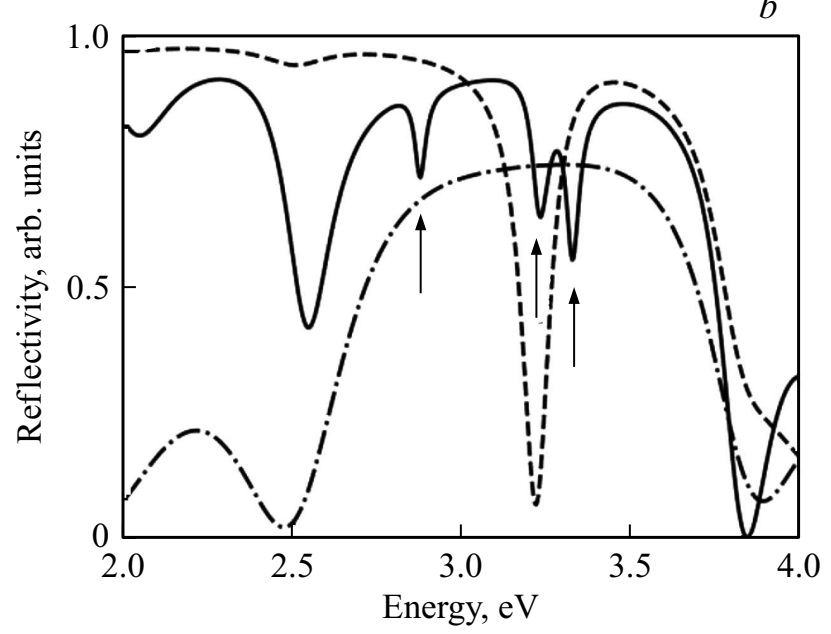

Рис. 1. $a-$ схема микрорезонатора с внутриметаллическими слоями и органической активной областью. Распределение электрического поля гибридной моды микрорезонатора (сплошная линия) и моды таммовского плазмона составной части структуры (штриховая линия). $b-$ спектры отражения от структуры с внутрирезонаторными слоями (сплошная линия) для случая $d_{\text {cav }}=70$ нм, структуры с таммовским плазмоном (штриховая линия) и распределенного брэгговского отражателя (штрихпунктирная линия).

Схема структуры показана на рис. $1, a$. В данном микрорезонаторе, при условии близости частот, имеет место гибридизация трех собственных мод: двух мод таммовского плазмона (ТР), локализованных между брэгговскими зеркалами и слоями металла и моды Фабри-Перо (FP), локализованной между двумя металлическими зеркалами $[11,16]$. Примеры распределения электрического поля гибридной моды и моды таммовского плазмона показаны на рис. $1, a$ сплошной и пунктирной линиями соответственно.

Спектр отражения от микрорезонатора с внутрирезонаторными слоями для случая $d_{\mathrm{cav}}=70$ нм показан на рис. $1, b$ сплошной линией. Также для сравнения показан спектр отражения от структуры с таммовским плазмоном (штриховая линия) и $\mathrm{Ta}_{2} \mathrm{O}_{5} / \mathrm{SiO}_{2}$ брэгговского отражателя (штрихпунктирная линия). Как было продемонстрировано в работе [16], ширины провалов, соответствующих каждой из гибридных мод, ниже, чем ширина провала, соответствующего таммовскому плазмону, что соответствует меньшему затуханию моды.

Распределение электрического поля и спектральное положение гибридных мод рассматриваемой системы может варьироваться в зависимости от толщины центрального слоя $\left(d_{\text {cav }}\right)$ и толщины двух фазосогласующих слоев $\left(d_{\mathrm{ph}}\right)$. В данной работе в качестве материала фазосогласующих слоев также используется материал центральной полости - СВР. Для анализа модовой структуры исследуемой системы был проведен расчет спектров отражения от структуры для фиксированного параметра $d_{\mathrm{ph}}=26$ нм и различных толщин центральной полости $d_{\text {cav. }}$ Полученные из аппроксимации спектров положения пиков, соответствующих собственным модам, показаны на рис. 2. Можно наблюдать 4 собственные моды. Можно видеть, что взаимодействие моды Фабри-Перо, энергия которой линейно зависит от размера полости с модой таммовского плазмона $\left(\hbar \omega_{T P} \approx 3.21 \mathrm{\jmath} \mathrm{B}\right)$, имеет характер антипересечения. Распределение квадрата электрического поля $\left(\left|\tilde{\epsilon}^{(1)}\right|^{2}+\left|\tilde{\epsilon}^{(2)}\right|^{2}\right)$ для каждой из четырех собственных мод, рассчитанных с помощью метода $S$-квантования $[8,17]$, также показано на рис. 2 . Можно выделить две моды (моды $a$ и $c$ ), которые характеризуются высокой степенью локализации в структуре, с максимумом поля в центральном слое. Взаимодействия излучателя, помещенного в центр активной области, с данными состояниями должно приводить к значительной модификации времени жизни возбужденного состояния (эффект Парселла).

С помощью формализма $S$-квантования $[8,17]$ были рассчитаны зависимости модового фактора Парселла для диполя, помещенного в центр активной полости, для различных толщин полости $\left(d_{\text {cav }}\right)$ в случае TE-поляризации и нормального угла падения. Результат расчета представлен на рис. 3. Можно видеть, что в областях, соответствующих дисперсии состояний, локализованных в центральном слое, наблюдается увеличение скорости спонтанной эмиссии. Причем максимальное значение модового фактора Парселла $(\sim 10)$ соответствует областям антипересечения таммовского плазмона с модой Фабри-Перо.

Стоит отметить, что во всех предыдущих результатах диэлектрическая проницаемость материала СВР была взята за постоянное значение $\left(\varepsilon_{b}=n_{b}^{2}\right)$. Наличие мощного экситонного резонанса может приводить к значительной перестройке картины люминесценции структуры. Сравнение результатов расчета модового фактора Парселла для случая без экситонного вклада $\left(\varepsilon_{\mathrm{CBP}}(\omega)=\varepsilon_{b}\right)$ и для случая с экситонным вкладом показано на рис. 4. Диэлектрическая проницаемость СВР 

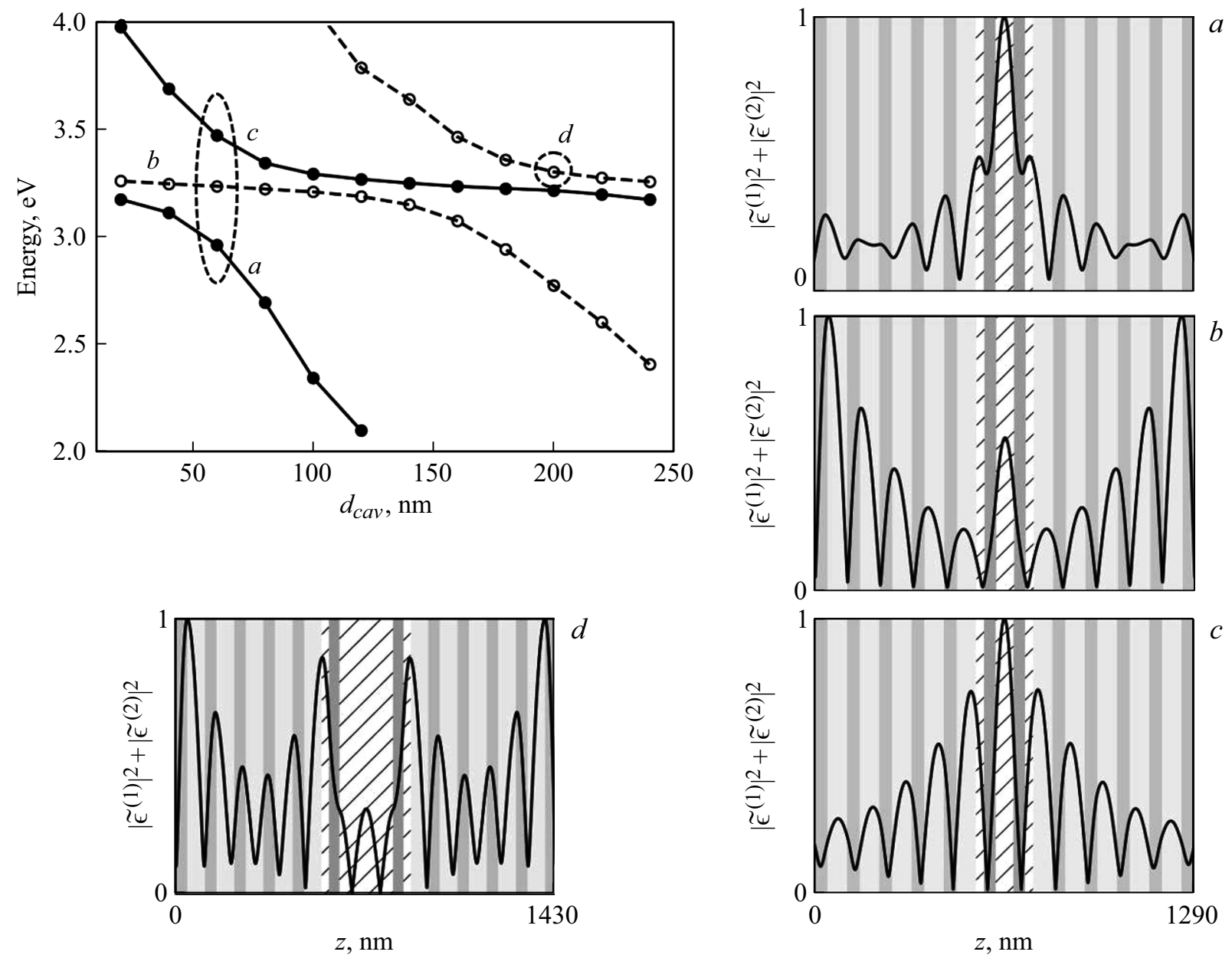

Рис. 2. Спектральное положение гибридных мод микрорезонатора в зависимости от размера центральной полости $\left(d_{\text {саv }}\right)$. Черные кружки и сплошные линии демонстрируют положение мод, сильно локализованных в центральном слое микрорезонатора. Распределение электрического поля собственных мод в случае $d_{\text {cav }}=60$ нм $(a, b, c)$ и $d_{\text {cav }}=200$ нм $(d)$ показано на рисунках $a-d$. На фоне показана схема слоев исследуемых структур.

с экситонным вкладом определяется выражением

$$
\varepsilon_{\mathrm{CBP}}(\omega)=\varepsilon_{b}+\frac{\omega_{L T}}{\omega_{\mathrm{exc}}-\omega-i \gamma_{\mathrm{exc}}}
$$

где $\varepsilon_{b}-$ фоновая диэлектрическая проницаемость (3.2), $\omega_{L T}$ - продольно-поперечное расщепление (130 мэВ), $\omega_{\text {exс }}$ - резонансная частота $(3.5$ эВ $)$ и $\gamma_{\text {ехс }}$ - нерадиационное затухания экситона (130 мэВ) [18]. Зависимость $\sqrt{\varepsilon_{\mathrm{CBP}}}(\omega)$ представлена на рис. $4, a$. На рис. $4, b$ и $c$ показана зависимость модового фактора Парселла от энергии и угла для структуры с $d_{\text {cav }}=60 \mathrm{Hм}$, при отсутствии экситонного вклада (рис. $4, b$ ) в диэлектрической проницаемости и при ее наличии (рис. $4, b$ ) для случая ТЕ-поляризации и эмиттера, помещенного в центр активной области. Белая линия и кружки показывают дисперсию собственных мод структуры. Можно видеть, что при отсутствии экситонного вклада в области частот и углов эмиссии, соответствующих двум гибридным модам, вероятность спонтанной эмиссии увеличена.

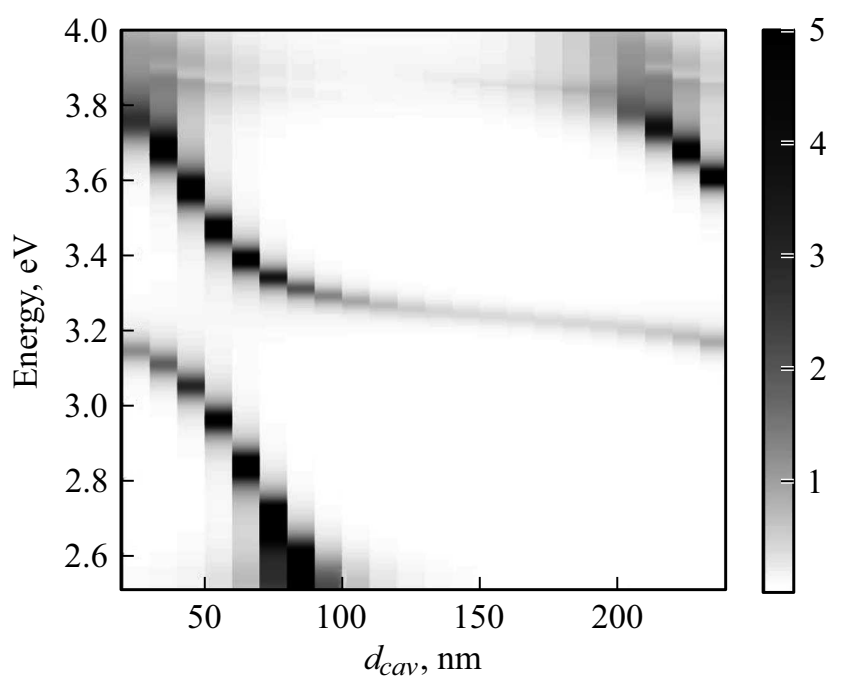

Рис. 3. Распределение модового фактора Парселла для ТЕ-поляризации и нормального падения света в случае диполя, помещенного в центр органической активной области в зависимости от размера центральной полости $\left(d_{\mathrm{cav}}\right)$. 


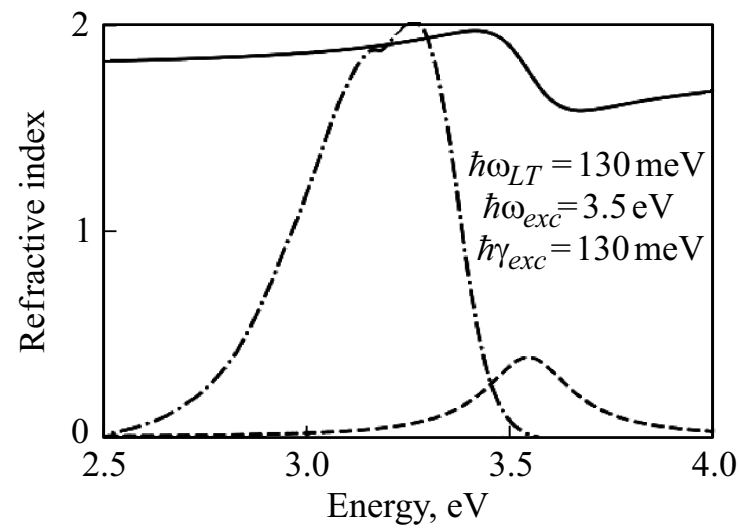

$a$
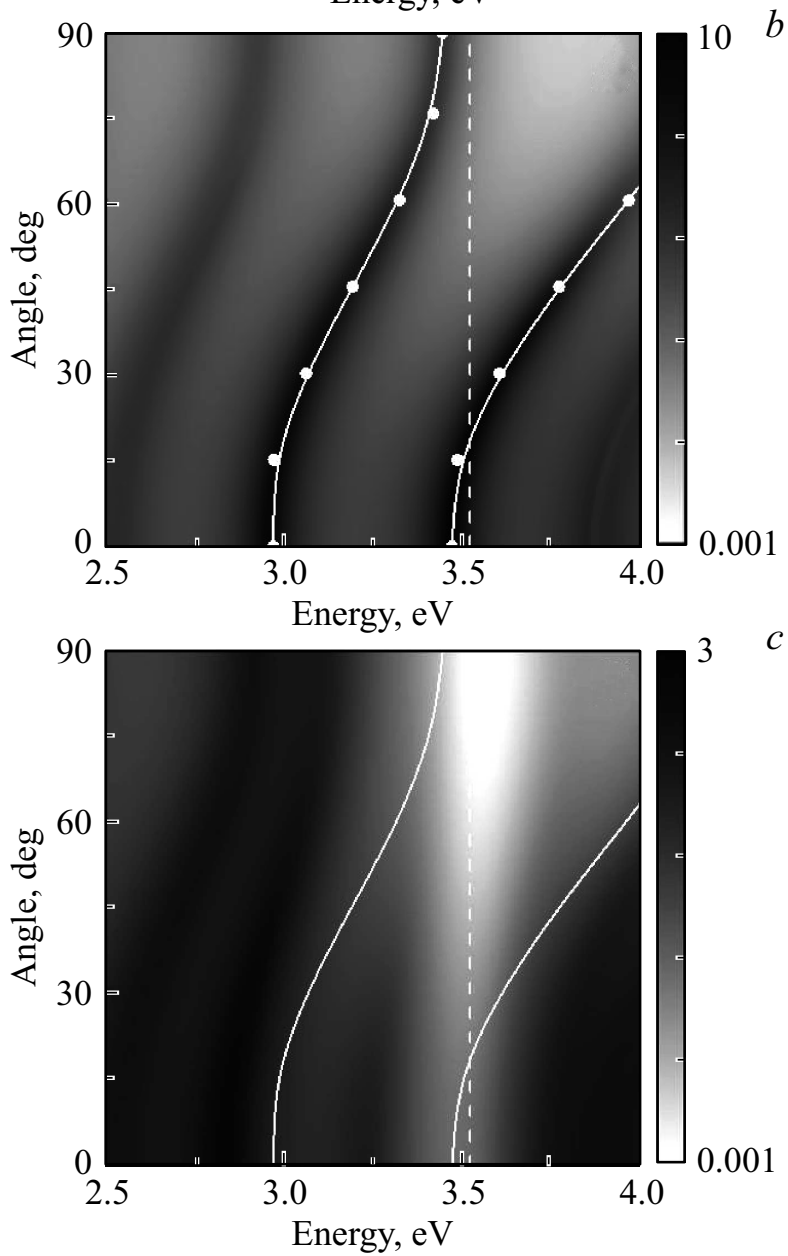

Рис. 4. $a-$ действительная (сплошная линия) и мнимая (пунктирная линия) части показателя преломления органического материала CBP (4, 4'-Bis ( $N$-carbazolyl)-1, $1^{\prime}$-biphenyl). Штрихпунктирная линия показывает спектр излучения материала. $b-$ рассчитанная зависимость модового фактора Парселла от энергии и угла для исследуемой структуры $\left(d_{\text {cav }}=60\right.$ нм $)$, при отсутствии экситонного вклада в диэлектрической проницаемости органического материала в случае ТЕ-поляризации и эмиттера, помещенного в центр активной области. Белая линия и кружки показывают дисперсию гибридных мод. $c-$ рассчитанная зависимость модового фактора Парселла от энергии и угла для гибридного резонатора при наличии экситона. Пунктирная линия показывает энергию экситонного резонанса.
При взаимодействии гибридных состояний с мощным экситоном в СВР имеет место уширение и сдвиг ветвей собственных мод (областей с увеличенной вероятностью спонтанной эмиссии) в низкоэнергетичную область спектра на величину $\sim 200$ мэВ, что может свидетельствовать о формировании режима сильной связи.

\section{3. Заключение}

Был проведен теоретический анализ возможности использования микрорезонаторной структуры с внутрирезонаторными металлическими слоями для усиления излучения органического материала (малой молекулы CBP $\left(4,4^{\prime}\right.$-Bis $(N$-carbazolyl)-1,1'-bipheny $\left.)\right)$ с широкой полосой излучения (от 2.5 до 3.5 эВ). Были проведены расчеты коэффициента отражения и вероятности спонтанной эмиссии от частоты и направления эмиссии для различных толщин активной области. Были исследованы модовый состав и распределения электромагнитных полей в структуре.

Было показано, что гибридные моды, локализованные в центральном слое СВР, могут значительно увеличивать скорость затухания люминесценции излучателя посредством эффекта Парселла. Также был обнаружен сдвиг гибридных мод при их взаимодействии с экситоном в СВР, что в свою очередь может означать появление режима сильной связи экситона и гибридной моды.

\section{Благодарность}

Авторы выражают благодарность Российскому научному фонду (проект № 16-12-10503).

\section{Конфликт интересов}

Авторы заявляют, что у них нет конфликта интересов.

\section{Список литературы}

[1] A.J.C. Kuehne, M.C. Gather. Chem. Rev., 116 (21), 12823 (2016).

[2] J.-H. Lee, C.-H. Chen, P.-H. Lee, H.-Y. Lin, M. Leung, T.-L. Chiu, C.-F. Lin. J. Mater. Chem. C, 7, 5874 (2019).

[3] R. Brückner, A.A. Zakhidov, R. Scholz, M. Sudzius, S.I. Hintschich, H. Früb, V.G. Lyssenko, K. Leo. Nature Photonics, $6(5), 322$ (2012).

[4] C. Symonds, G. Lheureux, J.P. Hugonin, J.J. Greffet, J. Laverdant, G. Brucoli, A. Lemaitre, P. Senellart, J. Bellessa. Nano Lett., 13 (7), 3179 (2013).

[5] S.-G. Huang, K.-P. Chen, S.-C. Jeng. Optical Mater. Express, 7 (4), 1267 (2017).

[6] R. Bikbaev, S. Vetrov I. Timofeev. Photonics, 5 (3), 22 (2018).

[7] S. V. Boriskina, Y. Tsurimaki. J. Phys.: Condens. Matter, 30 (22), 224003 (2018). 
[8] A.R. Gubaydullin, C. Symonds, J. Bellessa, K.A. Ivanov, E.D. Kolykhalova, M.E. Sasin, A. Lemaitre, P. Senellart, G. Pozina, M.A. Kaliteevski. Scientific Reports, 7, 9014 (2017).

[9] M.A. Kaliteevski, A.A. Lazarenko. Techn. Phys. Lett., 39, 698 (2013).

[10] R. Brückner, V.G. Lyssenko, S. Hofmann, K. Leo. Faraday Discuss, 174, 183 (2014).

[11] M.A. Kaliteevski, A.A. Lazarenko, N.D. Il'inskaya, Yu.M. Zadiranov, M.E. Sasin. D. Zaitsev, V.A. Mazlin, P.N. Brunkov, S.I. Pavlov, A.Yu. Egorov. Plasmonics, 10 (2), 281 (2015).

[12] D.G. Lidzey, D.D.C. Bradley, M.S. Skolnick, T. Virgili, S. Walker, D.M. Whittaker. Nature, 395, 53 (1998).

[13] D. Ballarini, M. De Giorgi, S. Gambino, G.Lerario, M. Mazzeo, A. Genco, G. Accorsi, C. Giansante, S. Colella, S. D’Agostino, P. Cazzato, D. Sanvitto, G. Gigli. Adv. Optical Mater., 2, 1076 (2014).

[14] V. Jankus, C. Winscom, A.P. Monkman. J. Chem. Phys., 130, 074501 (2009).

[15] K.M. Morozov, K.A. Ivanov, D. de Sa Pereira, C. Menelaou, A.P. Monkman, G. Pozina, M.A. Kaliteevski. Sci. Rep., 9, 9604 (2019).

[16] A.A. Lazarenko, K.A. Ivanov, A.R. Gubaydullin, M.A. Kaliteevski. Semiconductors, 51, 520 (2017).

[17] K.M. Morozov, E.I. Girshova, A.R. Gubaydullin, K.A. Ivanov, G. Pozina, M.A. Kaliteevski. J. Phys.: Condens. Matter, 30, 435304 (2018).

[18] B. Deveaud. The Physics of Semiconductor Microcavities: From Fundamentals to Nanoscale Devices (Wiley-VCH Verlag GmbH \& Co., 2007).

Редактор Г.А. Оганесян

\title{
Properties of Tamm plasmon-based microcavity with intracavity metallic layers and organic active region
}

\author{
K.M. Morozov' ${ }^{1}$, A.V. Belonovskii, ${ }^{1,2}$, E.I. Girshova1, \\ K.A. Ivanov ${ }^{2}$, M.A. Kaliteevski ${ }^{1,2,3}$ \\ ${ }^{1}$ St.Petersburg Academic University, \\ 194021 St. Petersburg, Russia \\ 2 ITMO Univesity, \\ 197101 St. Petersburg, Russia \\ ${ }^{3}$ loffe Institute, \\ 194021 St. Petersburg, Russia
}

Abstract A theoretical study of the properties of a microcavity structure with intracavity metal layers and an organic active region (material CBP $\left(4,4^{\prime}\right.$-Bis $\left(N\right.$-carbazolyl)-1, $1^{\prime}$-biphenyl $)$ ) was done. The structure consisted of a layer of organic material CBP, sandwiched between two silver layers, thin phase matching CBP layers $\left(d_{\mathrm{ph}}=26 \mathrm{~nm}\right)$ and two quarter-wave Bragg reflectors. It was shown that hybrid modes localized in the central layer of the structure can significantly increase the decay rate of the luminescence of an organic emitter by means of the Purcell effect. A shift in the hybrid modes spectral position was shown upon their interaction with the exciton resonance in CBP. This fact may indicate the appearance of a strong coupling regime. 